\title{
How to Untie Your Tongue COMMUNICATION CORNER
}

\author{
by Philip Yaffe
}

\section{Editor's Introduction}

Each "Communication Corner" essay is self-contained; however, they build on each other. For best results, before reading this essay and doing the exercise, go to the first essay "How an Ugly Duckling Became a Swan," then read each succeeding essay.

Communication is not only the written word. In this installment, Philip Yaffe shares tips and exercises that will help improve your skills in both writing and speaking. 


\title{
How to Untie Your Tongue
}

\author{
COMMUNICATION CORNER
}

\section{by Philip Yaffe}

In my book, The Gettysburg Approach to Writing \& Speaking like a Profession, I argue writing and speaking are so closely related that it is detrimental to treat them as separate disciplines. While they are built on the same foundations, writing and speaking have their own unique characteristics. By understanding the similarities and exploiting the differences, you can do both better.

If a picture is worth a thousand words, so is an example. Below is an article I wrote on a particular aspect of oral presentations. First read the article, next read how it was analyzed to convert it into a speech, and finally read the speech. I hope you will agree the article and the speech convey the same information, but in ways proper to their own particular characteristics.

You will notice the speech is shorter than the article for two reasons:

- It contains fewer transitional statements, because certain transitions can be better handled through body language and vocal intonation.

- It contains slightly less information, because sometimes information that reinforces a text can became distracting in a speech.

\section{Written Article}

According to studies, the hardest challenge for a novice public speaker is to get used to the idea of standing up in front of an audience. The second hardest challenge is to speak to that audience fluently. In other words avoiding ums, ahs, ers, you knows, and all the other so-called "filler words."

I used to be one of the worst offenders in stumbling over what I wanted to say. However the problem was cured almost overnight. How? I made a simple, almost obvious discovery that I would like to share with you. 
Mark Twain famously said: "The difference between the right word and the almost right word is the difference between lightning and a lightning bug." Of course he was absolutely right-and partially wrong.

As every professional writer knows, choosing exactly the right words to convey their meaning is crucial, because words are all they have. Anything less than les mots justes (exactly the right words, as they say in French), will weaken the message. Therefore, it is worth the time and effort to find les mots justes. Indeed, the commitment to doing so is one of the key factors that distinguish professional writers from amateurs.

On the other hand, searching out les mots justes in public speaking can be seriously counterproductive.

We all know people who seem able to speak fluently and persuasively about virtually any subject on a moment's notice. Likewise, we all know people who constantly trip over their tongues and appear bewildered even when speaking about subjects they know very well.

What makes the difference? I believe it is an unnecessary and fruitless search for les mots justes.

While using exactly the right word is always recommended, readers and listeners differ in how they process precise information. With a printed text, if people don't understand something, they have the luxury of reading it again. However, if they hear something they don't understand, it's there, then it's gone. End of story.

This is not a bad thing, because it means that listeners are less critical than readers. They are looking to take away broad general ideas. Details in the speech serve to define and defend these general ideas. They are not to be memorized for later examination.

Additionally, listeners more easily accept the validity of a general statement with less supporting evidence than they would require in a text. Thus, while a bit of imprecision may cause a slight blip in the listener's attention, it will quickly disappear, because neither the speaker nor the listener is in a position to dwell on it.

In short, when you write, take the time and trouble to always use the right word, but when you speak don't become obsessive about it. For any subject, there are usually several ways of saying the same thing. If you are always looking for the "best way" (assuming there is one), then you will invariably find yourself inserting er, um, ah, you know, and other distracting noises. In the vast majority of cases, sacrificing fluency while searching for les mots justes just isn't worth it. So go with what you have. 
But what if something comes out of your mouth that you think could have been said better? No problem. Simply start your next sentence with, "To be more precise . . . " or "To be absolutely clear . . .", then say it better. This technique will not only keep your speech fluent, it will make you appear to be master of your subject, rather than its apprentice.

What could be better than that?

\section{Analysis for Conversion to Speech}

According to studies, the hardest challenge for a novice public speaker is to get used to the idea of standing up in front of an audience. The second hardest challenge is to speak to that audience fluently. In other words avoiding ums, ahs, ers, you knows, and all the other so-called "filler words." [Demonstrate the problem by saying the preceding sentence with stumbles and hesitations.]

\section{[Insert transition that directly addresses the listening audience.]}

I used to one of the worst offenders in stumbling over what I wanted to say. However the problem was cured almost overnight. How? I made a simple, almost obvious discovery that I would like to share with you [here tonight].

Mark Twain famously said: "The difference between the right word and the almost right word is the difference between lightning and a lightning bug."

Of course, he was absolutely write-and partially wrong [Delete the preceding sentence.] As every professional writer knows, choosing exactly the right words to convey their meaning is crucial, because words are all they have. Anything less than les mots justes (exactly the right words, as they say in French), will weaken the message. [For easier oral comprehension, use the term "exactly the right word" before introducing the term "les mots justes."] Therefore, it is worth the time and effort to find les mots justes.

On the other hand, searching out les mots justes in public speaking can be seriously counterproductive. 
We all know people who seem able to speak fluently and persuasively about virtually any subject on a moment's notice. Likewise, we all know people who constantly trip over their tongues and appear bewildered even when speaking about subjects they know very well.

What makes the difference? I believe it is an unnecessary and fruitless search for les mots justes.

While using exactly the right word is always recommended [Delete the preceding phrase.], readers and listeners differ in how they process precise information. With a printed text, if people don't understand something, they have the luxury of reading it again. However, if they hear something they don't understand, it's there, then it's gone. End of story.

This is not a bad thing, because it means that listeners are less critical than readers. They are looking to take away broad general ideas. Details in the speech serve to define and defend these general ideas. They are not to be memorized for later examination.

Additionally, listeners more easily accept the validity of a general statement with less supporting evidence than they would require in a text. Thus, while a bit of imprecision may cause a slight blip in the listener's attention, it will quickly disappear, because neither the speaker nor the listener is in a position to dwell on it.

In short, when you write take the time and trouble to always use the right word, but when you speak don't become obsessive about it. [Delete the preceding sentence.] For any subject, there are usually several ways of saying the same thing. If you are always looking for the "best way" (assuming there is one), then you will invariably find yourself inserting er, um, ah, you know, and other distracting noises. In the vast majority of cases, sacrificing fluency while searching for les mots justes just isn't worth it. So go with what you have.

But what if something comes out of your mouth that you think could have been said better? No problem. Simply start your next sentence with, "To be more precise . . . " or "To be absolutely clear . . .", then say it better. This technique will not only keep your speech fluent, it will make you appear to be master of your subject, rather than its apprentice.

What could be better than that? [Add a more personal conclusion.] 


\section{Speech}

According to studies, the hardest challenge for a novice public speaker is to get used to the idea of standing up in front of an audience. The second hardest challenge is to speak to that audience fluently. In other words avoiding ums, ahs, ers, you knows, and all the other so-called filler words. [Say the preceding sentence with stumbles and hesitations.]

If you fear this problem, I have some good news for you. I used to one of the worst offenders in stumbling over what I wanted to say. However the problem was cured almost overnight. How? I made a simple, almost obvious discovery that I would like to share with you here tonight.

Mark Twain famously said: "The difference between the right word and the almost right word is the difference between lightning and a lightning bug."

As every professional writer knows, choosing exactly the right words to convey their meaning is crucial, because words are all they have. Anything less than les mots justes (exactly the right words, as they say in French), will weaken the message. Therefore, it is worth the time and effort to look for them.

On the other hand, searching out les mots justes in public speaking can be seriously counterproductive.

We all know people who seem able to speak fluently and persuasively about virtually any subject on a moment's notice. Likewise, we all know people who constantly trip over their tongues and appear bewildered even when speaking about subjects they know very well.

What makes the difference? I believe it is an unnecessary and fruitless search for les mots justes.

Readers and listeners differ in how they process precise information. With a printed text, if people don't understand something, they have the luxury of reading it again. However, if they hear something they don't understand, it's there, then it's gone. End of story.

This is not a bad thing, because it means that listeners are less critical than readers. They are looking to take away broad general ideas. Details in the speech serve to define and defend these general ideas. They are not to be memorized for later examination.

Thus, while a bit of imprecision may cause a slight blip in the listener's attention, it will quickly disappear because neither the speaker nor the listener is in a position to dwell on it. 
In short, when you write take the time and trouble to always use the right word, but when you speak don't become obsessive about it. For any subject, there are usually several ways of saying the same thing. If you are always looking for the "best way" (assuming there is one), then you will invariably find yourself inserting er, um, ah, you know, and other distracting noises. In the vast majority of cases, sacrificing fluency while searching for les mots justes just isn't worth it. So go with what you have.

But what if something comes out of your mouth that you think could have been said better? No problem. Simply start your next sentence with, "To be more precise . . . " or "To be absolutely clear . . ." then say it better. This technique will not only keep your speech fluent, it will make you appear to be master of your subject, rather than its apprentice.

What could be better than that?

Hopefully I have now been speaking to you fluently for several minutes, so, um, I think ah, that I, ah, can now relax and, um, ah, speak more like... . Oh, the hell with it. Now that I know how to be fluent, speaking like I used to is just too damn hard.

\section{HOMEWORK: Retrospective to Communication Corner No. 10}

In the previous installment, you were asked to read a summary of everything you had already learned about expository (non-fiction) writing in the first nine essays in the Communication Corner series, and to fix all the key ideas firmly in mind. You were also asked to think about how the principles and practices of good writing might also be applied to giving a good speech or other types of oral presentations.

If you haven't already done so, please do so now. Understanding these key principles and practices will be crucial for fully benefiting from all the Communication Corner essays to follow.

\section{CURRENT HOMEWORK}

If conciseness is critical in writing, it is even more so (if that's possible) in speaking.

When you speak, your words flow over the listeners like a cloud. First they are there, then they are gone. If the listeners don't immediately grasp what you are saying, they have no opportunity to go back over a sentence to try to divine its meaning. Therefore, everything you 
say needs to advance your meaning. Using unnecessary words or using ambiguous words and phrases virtually ensures that listeners will not fully grasp your message.

Here are a few exercises to hone your conciseness skills in both writing and speaking. Remember the definition of conciseness: As long as necessary, as short as possible (Co $=\mathrm{LS})$.

These exercises generally contain all the information you will need. However, to write clearly and concisely, it is often necessary research key information that may be lacking. For the purposes of these exercises, if you feel some key information is lacking, you can simply make it up.

\section{How to Do These Exercises}

Each exercise has two texts labeled "Original" and "Analysis." The procedure is as follows:

1. Cover the page so that only the Original is visible and read the text.

2. As you read, and with everything else still covered, make notes about what you think needs to be done to improve the Original.

3. Uncover the Analysis and compare it with your notes.

4. Rewrite the text.

In the next installment (CC12), you be able to compare your revision of the text with my revised version. Remember: There is always more than one way of writing and saying things well. So if your revision doesn't match mine, don't be concerned. The important thing is that it is concise, i.e. as long as necessary: as short as possible (Co $=$ LS).

\section{Exercise 1}

[Original]

During employment with $A B C$ Corporation, employees are legally entitled to take leave from the office during normal working hours. When this leave is taken, employees continue to receive their normal salary.

[Analysis]

During employment with $\mathrm{ABC}$ Corporation [Unnecessary words], employees are legally entitled to take leave from work during normal working hours [Unnecessary words]. When this leave is taken [Unnecessary words], employees continue to receive their normal salary.

Write your revision. 


\section{Exercise 2}

[Original]

Annual leave entitlements are generated in accordance with legal requirements. Employees are credited with their annual leave entitlement during the working year and entitled to access this balance as from January 1 of the following year.

[Analysis]

Annual leave entitlements are generated in accordance with legal requirements [ $\boldsymbol{s}$ this sentence necessary?]. Employees are credited with their annual leave entitlement during the working year and entitled to access this balance as from January 1 of the following year [Not clear; be more explicit].

Write your revision.

\section{Exercise 3}

[Original]

An employee may accumulate annual leave beyond the legal minimum only in the following circumstances:

1. If there is written documentation proving that the employee's immediate manager has refused a leave request.

2. If there is written proof that there were special circumstances why annual leave couldn't be taken during the current year.

3. If the employee's immediate manager otherwise supports an application for additional leave

[Analysis]

An employee may accumulate annual leave beyond the legal minimum [What is this minimum?] only in the following circumstances.

1. If there is written documentation [Use active voice] proving that the employee's immediate manager refused a leave request. 
2. If there is written proof [Use active voice] that there were special circumstances why annual leave couldn't be taken during the current year.

3. If the employee's immediate manager otherwise supports an application for additional leave.

Avoid repetition of "If" at the beginning of each bullet point.

Write your revision.

\section{Exercise 4}

[Original]

BBX is an agency born with the Swedish Internet, in 1996. Established in Stockholm, it has seen rapid growth and has attained the status of a completely multi-disciplinary, integrated agency.

The skills of the agency have indeed developed rapidly. This enables it to offer an extremely wide range of services today, with the same concern for quality and professionalism. These range from interface design to brand building, from Internet strategy and loyalty magazines to training in client satisfaction, not forgetting the more classic aspects of above and below the line marketing: direct mail, address list management, media planning . . . all while keeping an important point in mind; increasing the quality of the relationship which our clients maintain with their prospects or their consumers.

[Analysis]

BBX is an agency [Unnecessary words] born with the Swedish Internet, in 1996 [Move date]. Established in Stockholm, it has seen rapid growth and has attained the status of a completely multi-disciplinary, integrated agency.

The skills of the agency have indeed developed rapidly [Is this sentence necessary?]. This enables it to offer an extremely wide range of services today, with the same [Same as what?] concern for quality and professionalism. These [Services?] range from interface design to brand building, from Internet strategy and loyalty magazines to training in client satisfaction, not forgetting the more classic aspects of above and below the line marketing: direct mail, address list management, media planning . . . all while keeping an important point in mind; increasing the quality of the relationship which our 
clients maintain with their prospects or their consumers. [The sentence is too complex; services not easily distinguishable. Simplify.]

Write your revision.

\section{About the Author}

Philip Yaffe was born in Boston, Massachusetts, in 1942 and grew up in Los Angeles, where he graduated from the University of California with a degree in mathematics and physics. In his senior year, he was also editor-in-chief of the Daily Bruin, UCLA's daily student newspaper. He has more than 40 years of experience in journalism and international marketing communication. At various points in his career, he has been a teacher of journalism, a reporter/feature writer with The Wall Street Journal, an account executive with a major international press relations agency, European marketing communication director with two major international companies, and a founding partner of a specialized marketing communication agency in Brussels, Belgium, where he has lived since 1974. He is the author of more than 20 books, which can be found easily in Amazon Kindle.

DOI: $10.1145 / 3298983$ 